Article

\title{
Thermal Decomposition of Maya Blue: Extraction of Indigo Thermal Decomposition Steps from a Multistep Heterogeneous Reaction Using a Kinetic Deconvolution Analysis
}

\author{
Yui Yamamoto and Nobuyoshi Koga * \\ Chemistry Laboratory, Department of Science Education, Graduate School of Education, Hiroshima University, \\ 1-1-1 Kagamiyama, Higashi-Hiroshima 739-8524, Japan \\ * Correspondence: nkoga@hiroshima-u.ac.jp; Tel.: +81-82-424-7092
}

Academic Editor: Sergey Vyazovkin

Received: 2 June 2019; Accepted: 6 July 2019; Published: 9 July 2019

check for updates

\begin{abstract}
Examining the kinetics of solids' thermal decomposition with multiple overlapping steps is of growing interest in many fields, including materials science and engineering. Despite the difficulty of describing the kinetics for complex reaction processes constrained by physico-geometrical features, the kinetic deconvolution analysis (KDA) based on a cumulative kinetic equation is one practical method of obtaining the fundamental information needed to interpret detailed kinetic features. This article reports the application of KDA to thermal decomposition of clay minerals and indigo-clay mineral hybrid compounds, known as Maya blue, from ancient Mayan civilization. Maya blue samples were prepared by heating solid mixtures of indigo and clay minerals (palygorskite and sepiolite), followed by purification. The multistep thermal decomposition processes of the clay minerals and Maya blue samples were analyzed kinetically in a stepwise manner through preliminary kinetic analyses based on a conventional isoconversional method and mathematical peak deconvolution to finally attain the KDA. By comparing the results of KDA for the thermal decomposition processes of the clay minerals and the Maya blue samples, information about the thermal decomposition steps of the indigo incorporated into the Maya blue samples was extracted. The thermal stability of Maya blue samples was interpreted through the kinetic characterization of the extracted indigo decomposition steps.
\end{abstract}

Keywords: Maya blue; indigo; palygorskite; sepiolite; thermal decomposition; kinetic deconvolution analysis

\section{Introduction}

Thermal decomposition of inorganic solids is a complex heterogeneous reaction that is regulated by chemical kinetics and physio-geometrical constraints [1-3]. In addition, consecutive or concurrent reaction steps that originated from both chemical [4-9] and physio-geometrical reaction mechanisms can occur [10-16], wherein the individual reaction steps may be kinetically dependent on one another. For a multistep process in a homogeneous system, the kinetic behavior can be formalized using concentrations of reactant and intermediate and each reaction step can be verified according to probability considerations. By contrast, in the heterogeneous system, the rigorous formalization of the kinetic equation for multistep reactions is not so easy because of the physico-geometrical constraints of each reaction step and these complex interactions [17]. When each reaction step that exhibits Arrhenius-type temperature dependence can be approximated to be kinetically independent from the 
other steps, kinetic deconvolution analysis (KDA) can be applied to find an empirical solution for the kinetic description of multistep solid-state reactions [18,19], as follows:

$$
\frac{\mathrm{d} \alpha}{\mathrm{d} t}=\sum_{i=1}^{N} c_{i} A_{i} \exp \left(-\frac{E_{\mathrm{a}, i}}{R T}\right) f_{i}\left(\alpha_{i}\right) \text { with } \sum_{i=1}^{N} c_{i}=1 \text { and } \sum_{i=1}^{N} c_{i} \alpha_{i}=\alpha
$$

where $\alpha, c, A, E_{\mathrm{a}}$, and $R$ are the fractional reaction, contribution, Arrhenius pre-exponential factor, apparent activation energy, and the gas constant, respectively. The subscript $i$ denotes a reaction step out of a total $N$ steps. The function $f(\alpha)$ describes the physico-geometrical reaction mechanism as formalized by considering the rate-limiting step of the reaction and the reaction geometry [1-3]. Despite the empirical nature of the kinetic analysis using KDA, the results provide necessary information to gain further insights into consecutive or concurrent kinetic features [20-22], as well as practically useful information about the multistep process, including the contribution $\left(c_{i}\right)$ and apparent kinetic parameters $\left(A_{i}, E_{\mathrm{a}, i}\right.$, and $\left.f_{i}\left(\alpha_{i}\right)\right)$ of each reaction step $i$. Using the results of KDA, the overall reaction process, under a specific heating condition, can be reproduced or simulated. By comparing the results of KDA among a series of samples and under different reaction conditions, characteristics of multistep kinetic behavior can be correlated to different components of a composite sample [23-26] and specific reaction conditions $[27,28]$. KDA is also used to extract kinetic information about a selected reaction step from the overall process $[29,30]$.

A multistep heterogeneous thermal decomposition can be observed for inorganic-organic hybrid materials. One example of such a material is Maya blue (MB), a well-known pigment used in the Mayan civilization. MB is a hybrid compound of a microporous clay mineral and indigo [31-33]. Palygorskite and sepiolite are the typical clay minerals used in the preparation of MB. These fibrous clay minerals exhibit external and internal nanochannels, which are typically filled with zeolitic water [34-41]. MB is produced by the replacement of the zeolitic water with indigo molecules [31,34-36]. Notably, MB exhibits high stabilities against thermal treatment, light exposure, and acid and/or base attacks. Consequently, the structural characteristics of MB have been intensively studied using spectroscopic techniques that include Fourier transform infrared (FT-IR) and Raman spectroscopies [31,34,35,41-44]. The thermal behavior of MB has also been studied using thermoanalytical techniques [34,36,42]. The formation of strong hydrogen bonds between the structural water of the clay mineral and the carbonyl and amino functional groups of the indigo molecules was reported as a possible reason for the high stabilities of MB [37]. MB with palygorskite as the clay mineral is expected to be more stable compared to $\mathrm{MB}$ with uses sepiolite, because of the higher number of hydrogen bonds available to form between the indigo and the substrate mineral [35]. It was also reported that the indigo molecules incorporated into the clay mineral substrate transform into dehydroindigo during the heating process used to prepare MB $[37,43,45]$. The thermal decomposition of MB begins just above room temperature and indicates partially overlapping multistep processes upon further heating, which may be composed of dehydration steps of zeolitic, coordinating, and structural waters; decomposition of hydroxides in the substrate mineral; and sublimation/decomposition of indigo molecules [34,36,42]. Indigo molecules that have been incorporated into the clay mineral are known to have a higher thermal stability, as confirmed by thermoanalytical curves, compared to pure indigo crystals [34].

Kinetic characterization of the thermal decomposition of MB is a promising approach to evaluating its thermal stability. However, its complex multistep thermal behavior interferes with a successful and straight-forward kinetic analysis. Application of KDA to the thermal decomposition of MB is one possible empirical method of separating the overlapping reaction steps and kinetically characterizing each one to determine the reaction steps that relate specifically to the thermal decomposition of indigo molecules. In the present study, MBs based on palygorskite (P-MB) and sepiolite (S-MB) were prepared by heating solid mixtures of indigo and clay minerals. The thermal decomposition processes of the purified clay mineral substrate samples and the MB samples were analyzed kinetically using KDA, after the necessary preliminary kinetic approaches. By comparing the kinetic results for the thermal 
decomposition of $\mathrm{MB}$ and its clay mineral substrates, the thermal decomposition steps of the indigo molecules were extracted. Using the kinetic information for the thermal decomposition steps of the indigo molecules, the kinetic stabilities of the indigo molecules incorporated in different clay mineral matrices were compared to one another and those of pure indigo crystals.

\section{Results and Discussion}

\subsection{Sample Preparation and Characterization}

The characterization details of the purchased palygorskite and sepiolite samples are described in Section S1(1) in the Supplementary Materials. Since a $\mathrm{CaCO}_{3}$ impurity was found in both clay mineral samples, they were purified using $\mathrm{HCl}(\mathrm{aq})$ before use. Figure 1 represents the scanning electron microscope (SEM) images of the clay minerals after treatment with $\mathrm{HCl}(\mathrm{aq})$. The palygorskite sample is an agglomerate of needle-like crystal with a length of approximately 2-5 $\mu \mathrm{m}$ (Figure 1a). Agglomerates of columnar crystals, with a length of approximately 1-2 $\mu \mathrm{m}$, are characteristic of the sepiolite sample (Figure 1b).

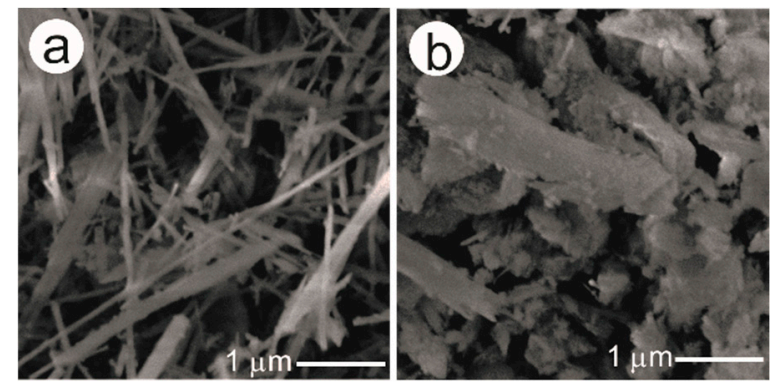

Figure 1. SEM images of the clay minerals after treatment with $\mathrm{HCl}(\mathrm{aq})$ : (a) Palygorskite and (b) sepiolite.

The coloration changes in the indigo-clay mineral mixture before and after heating are described in Section S1(2) in the Supplementary Materials. Figure 2 compares thermogravimetry (TG)-derivative thermogravimetry (DTG)-differential thermal analysis (DTA) curves for the heat-treated samples with different indigo/clay mineral ratios. The major difference between the samples with different indigo/clay mineral ratios is the mass-loss step initiated at approximately $525 \mathrm{~K}$ and $535 \mathrm{~K}$ for the palygorskite and sepiolite substrate samples, respectively, in which the mass-loss value and the DTG peak height increase with an increasing amount of indigo. The temperature range of the mass-loss step agrees with that for the sublimation/decomposition of pure indigo crystals (Figure S9). Therefore, it is likely that, in the samples with higher indigo/clay mineral ratios, excess indigo remains unreacted with the clay mineral substrates.

(a)

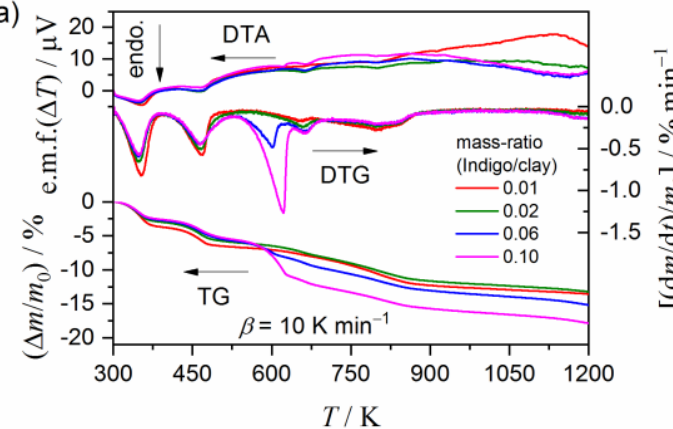

(b)

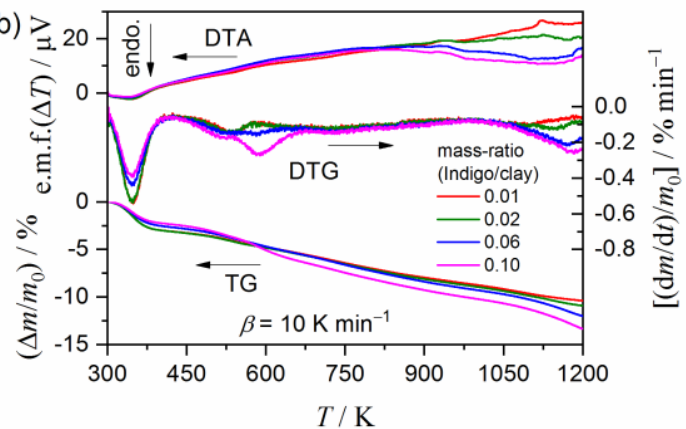

Figure 2. TG-DTG-DTA curves for the heat-treated indigo/clay mineral mixtures synthesized using different initial ratios: (a) Indigo/palygorskite and (b) indigo/sepiolite. 
Figure 3 compares the TG-DTG-DTA curves for the heat-treated indigo/palygorskite sample and those further treated with $\mathrm{Na}_{2} \mathrm{~S}_{2} \mathrm{O}_{4}$ (aq) for removing excess indigo. The TG-DTG-DTA curves were recorded under an atmosphere of flowing $\mathrm{N}_{2}$ (Figure $3 \mathrm{a}$ ) and clearly indicate that the mass-loss step initiated at approximately $525 \mathrm{~K}$ disappears after samples are treated with $\mathrm{Na}_{2} \mathrm{~S}_{2} \mathrm{O}_{4}(\mathrm{aq})$. For the sample treated with $\mathrm{Na}_{2} \mathrm{~S}_{2} \mathrm{O}_{4}(\mathrm{aq})$, several mass-loss steps that occur at the higher temperatures also disappeared in the TG-DTG-DTA curves recorded in flowing air (Figure 3b). The disappeared mass-loss steps are expected to be attributed to either oxidation or combustion of the thermal decomposition product of indigo. The comparable results of $\mathrm{Na}_{2} \mathrm{~S}_{2} \mathrm{O}_{4}(\mathrm{aq})$ treatment were also observed for the heat-treated indigo/sepiolite samples, as shown in Figure S10. These results indicate that the removal of excess indigo from the MB samples was successful. Figure S11 compares the sample coloration before and after the $\mathrm{Na}_{2} \mathrm{~S}_{2} \mathrm{O}_{4}(\mathrm{aq})$ treatment. The faded color that results after the treatment arises from the removal of excess indigo. The color fading is characterized by a decrease in the ultraviolet-visible (UV-Vis) absorption in the wavelength range of 425-600 $\mathrm{nm}$ and the appearance of a maximum absorption at approximately $650 \mathrm{~nm}$, as illustrated in Figure S12. Figure S13 presents the SEM images of the samples treated with $\mathrm{Na}_{2} \mathrm{~S}_{2} \mathrm{O}_{4}(\mathrm{aq})$. The appearance of the synthesized MB samples was not significantly different from those of the palygorskite and sepiolite samples (Figure 1).
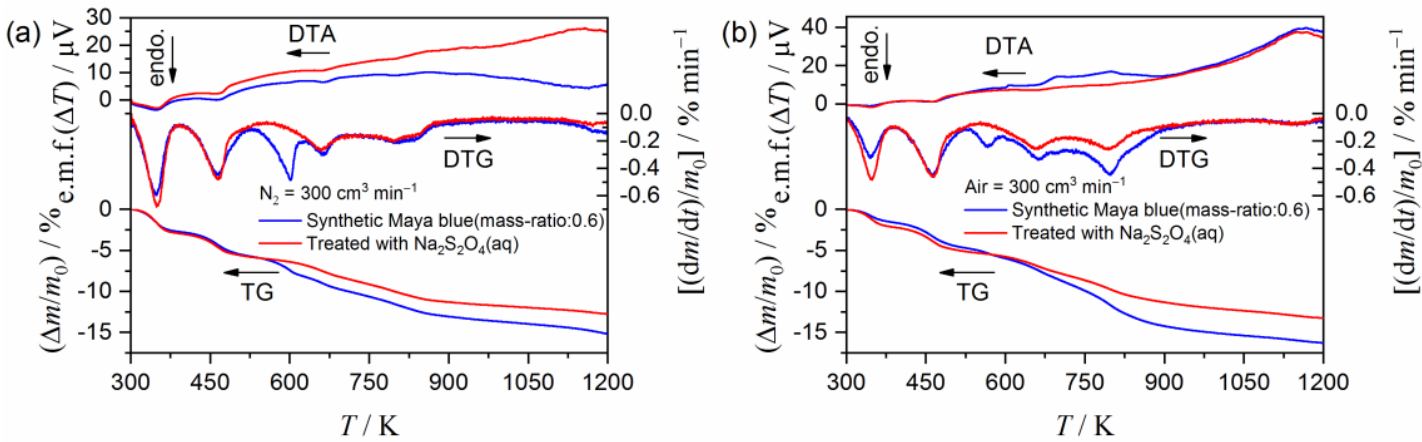

Figure 3. Comparison of the TG-DTG-DTA curves for the heat-treated indigo/palygorskite samples before and after treatment with $\mathrm{Na}_{2} \mathrm{~S}_{2} \mathrm{O}_{4}(\mathrm{aq})$ recorded in (a) flowing $\mathrm{N}_{2}$ and (b) flowing air.

\subsection{Kinetic Analysis of the Thermal Decomposition of Clay Minerals}

Figure 4 presents TG-DTG curves for the purified clay minerals, recorded at different heating rates $(\beta)$ in the flow of $\mathrm{N}_{2}$ gas. The thermal decomposition processes of both clay minerals are multistep processes comprised of three and four distinguishable DTG peaks for palygorskite (Figure 4a) and sepiolite (Figure $4 \mathrm{~b}$ ), respectively. The systematic shift of all the distinguishable DTG peaks to higher temperatures with increasing $\beta$ was observed for both samples. This is a normal feature for the kinetic process. The average value for the total mass loss during heating the samples to $1223 \mathrm{~K}$ was $13.6 \pm 0.3 \%$ and $9.9 \pm 0.1 \%$ for palygorskite and sepiolite, respectively. The overall thermal decomposition behaviors of palygorskite and sepiolite approximately agree with those previously reported $[34,46,47]$. The first to third DTG peaks in both the samples correspond to the thermal dehydration of zeolite water, the first coordinated water, and the second coordinated water. The fourth DTG peak in the thermal decomposition of sepiolite is attributed to the thermal dehydration of structural water. 

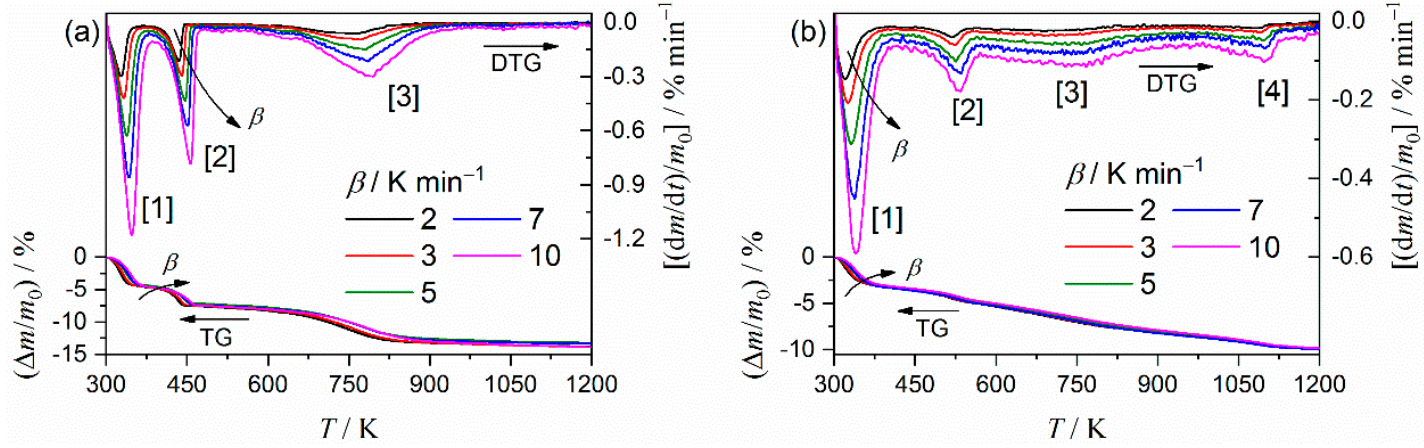

Figure 4. TG-DTG curves for the thermal decomposition of the purified clay minerals recorded at different $\beta$ in a flow of $\mathrm{N}_{2}$ gas: (a) Palygorskite and (b) sepiolite.

As part of the preliminary kinetic approach to the multistep thermal decomposition process, the isoconversional kinetic analysis was examined for the overall thermal decomposition. For the ideal single-step reaction, Equation (2) can be used as the fundamental kinetic equation [48].

$$
\frac{\mathrm{d} \alpha}{\mathrm{d} t}=A \exp \left(-\frac{E_{\mathrm{a}}}{R T}\right) f(\alpha)
$$

Taking the natural logarithm of both sides of Equation (2), one can obtain the following equation:

$$
\ln \left(\frac{\mathrm{d} \alpha}{\mathrm{d} t}\right)=\ln [A f(\alpha)]-\frac{E_{\mathrm{a}}}{R T}
$$

At the selected $\alpha$, the plot of the left-hand side of Equation (3) versus the reciprocal temperature should exhibit a linear correlation when the value of $\ln [A f(\alpha)]$ is constant. The apparent $E_{\mathrm{a}}$ values at different $\alpha$ can be calculated from the slope of the plot, known as a Friedman plot [49]. The application of the Friedman plot to the overall kinetic data of the multistep thermal decomposition process recorded at different $\beta$ is not supported by theory, because more than one reaction step overlaps at each $\alpha$. Even so, some possibility of finding $\alpha$ region characterized by a relevant $E_{\mathrm{a}}$ values or a specific trend of the $E_{\mathrm{a}}$ variation is still anticipated. Figure 5 illustrates the $E_{\mathrm{a}}$ values at different $\alpha$ for the overall thermal decomposition. For the thermal decomposition of palygorskite, four distinguishable reaction steps are expected from the constant $E_{\mathrm{a}}$ regions and the region that exhibits specific $E_{\mathrm{a}}$ variation trends (Figure 5a). The regions assigned as (1), (3), and (4) correspond to the major reaction steps observed as distinguishable DTG peaks. Five distinguishable $\alpha$ regions were found for the thermal decomposition of sepiolite (Figure 5b), in which the regions assigned as (1), (3), (4), and (5) correspond to the major DTG peaks.
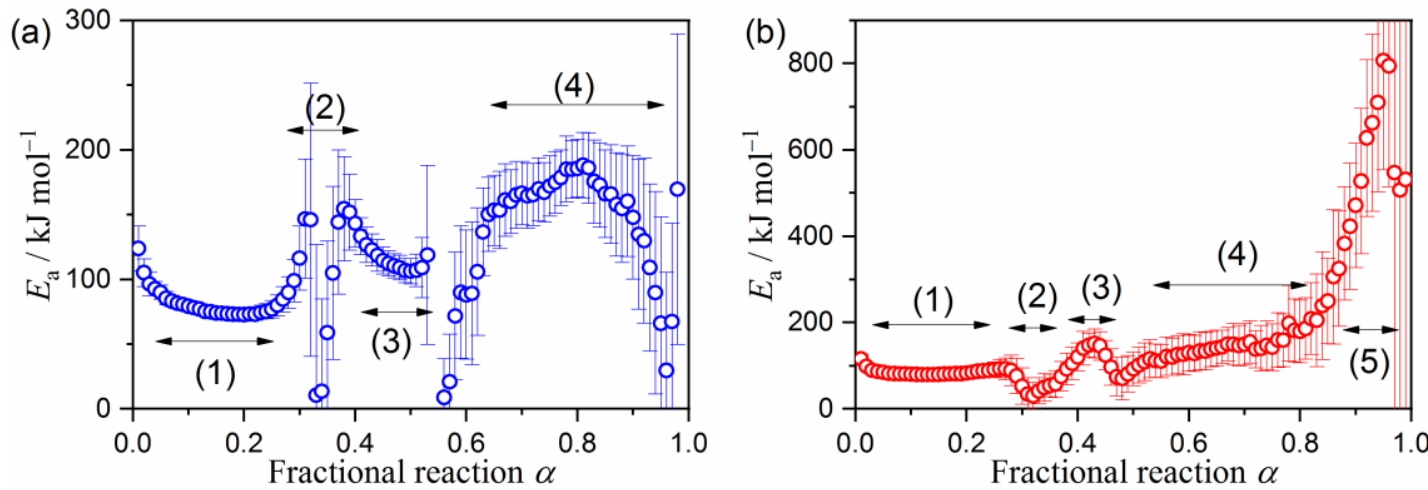

Figure 5. The apparent $E_{\mathrm{a}}$ values at different $\alpha$ with reference to the total mass-loss during the overall thermal decomposition of the purified clay minerals, determined by the Friedman plot: (a) Palygorskite and (b) sepiolite. 
Based on the results of the empirical application of the isoconversional method to the multistep thermal decomposition process, the component reaction steps were deconvolved based on the DTG curves by mathematical deconvolution analysis (MDA); that is, the statistical shape analysis assuming overlapping independent peaks are present $[18,50,51]$.

$$
\frac{\mathrm{d} m}{\mathrm{~d} t}=\sum_{i=1}^{N} F_{i}(t)
$$

In Equation (4), $N$ is the number of component peaks. The value $F_{i}(t)$ is the statistical function used to satisfactorily fit the component peak $i$. As the component DTG peaks typically have an asymmetric shape, one of the statistical functions that are applicable to symmetric peaks, such as the Weibull and Frazer-Suzuki functions, is favorable for MDA [18,50,51]. According to the number of distinguishable regions of $\alpha$ observed in the results of the isoconversional kinetic analysis (Figure 5), MDA for the thermal decomposition of palygorskite and sepiolite was carried out by setting $N=4$ and $N=5$, respectively. The Weibull function (Equation (S1)) was applied to fit all the component peaks.

Figure 6 illustrates a typical result of the MDA. The second DTG peaks in both samples are described by the partial overlapping of two peaks in the MDA results. The primary outcome from the MDA is the rough estimation of the contribution $c_{i}$ of each reaction step $i$ with reference to the overall reaction. Table S1 lists the contribution of each component step $i$. In both the samples, the first and fourth deconvolved steps, which correspond to the dehydration of zeolite water and the second coordinated water, have been indicated as significant contributions to the overall thermal decomposition.
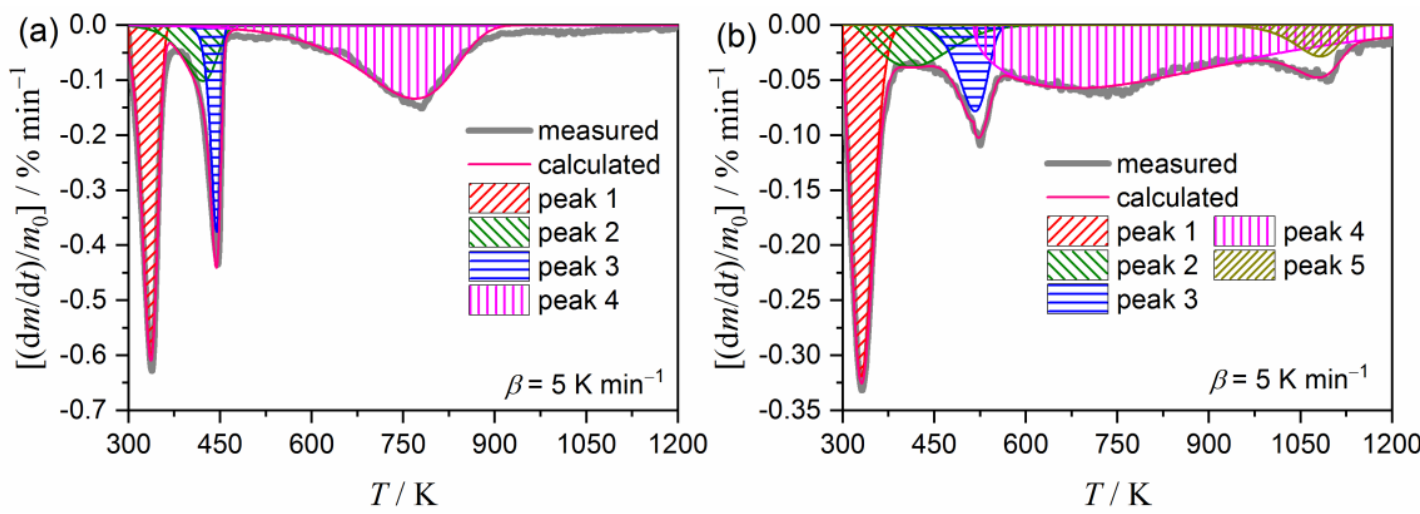

Figure 6. Typical results of MDA applied to the multistep thermal decomposition of the clay minerals: (a) Palygorskite and (b) sepiolite.

The other outcome from MDA is the separated kinetic curves for each reaction step. The features of the separated kinetic curves and the formal kinetic analyses for these kinetic curves are described in Section S2 in the Supplementary Materials.

Based on the results obtained by the preliminary kinetic approaches using the conventional isoconversional method and MDA, the overall kinetic curves were analyzed by assuming that the overlapping multistep process was comprised of independent reaction steps. In this case, the cumulative kinetic equation in Equation (1) is applicable [17-19]. For the kinetic model function $f_{i}\left(\alpha_{i}\right)$ for each reaction step $i$, an empirical kinetic model that accommodates different types of the physico-geometrical reaction mechanisms and those that deviate are needed to obtain the sophisticated fit for the calculated kinetic curve to the experimental kinetic curve. The Šesták-Berggren (SB) model with three kinetic exponents [52-54], $\mathrm{SB}(m, n, p)$, is one such empirical kinetic model with the high flexibility needed for the fitting, as follows:

$$
f(\alpha)=\alpha^{m}(1-\alpha)^{n}[-\ln (1-\alpha)]^{p}
$$


The nonlinear least squares analysis for fitting the calculated kinetic curve to the calculated kinetic curve while simultaneously optimizing all the kinetic parameters in Equations (1) and (5) is a typical procedure of KDA [17-19]. For reliable KDA, appropriate initial values of all the kinetic parameters that will be optimized through KDA are necessary. For the thermal decomposition of palygorskite and sepiolite, the initial $c_{i}$ and $E_{\mathrm{a}, i}$ values were adapted from the results of the MDA (Table S1). The initial kinetic exponents in the $\mathrm{SB}$ model were set to $\mathrm{SB}(0,1,0)$, which is the first-order kinetic model. Then, the order of $A_{i}$ values was determined graphically by monitoring the fit of the calculated kinetic curve to the experimental kinetic curve. After inputting all the initial values, KDA was run to optimize the values through nonlinear least squares analysis to minimize the sum of squares of the differences between the experimental and calculated kinetic curves, as follows:

$$
F=\sum_{j=1}^{M}\left[\left(\frac{\mathrm{d} \alpha}{\mathrm{d} t}\right)_{\exp , j}-\left(\frac{\mathrm{d} \alpha}{\mathrm{d} t}\right)_{\mathrm{cal}, j}\right]^{2}
$$

where $M$ is the total number of data points in the experimental kinetic curve at a $\beta$ value.

Figure 7 illustrates typical results of the KDA for the thermal decomposition of the purified palygorskite (Figure 7a) and sepiolite (Figure 7b). Regardless of the kinetic curve recorded at different $\beta$ values, the calculated kinetic curve was fit to the experimental kinetic curve with a determination coefficient for the nonlinear least squares analysis $\left(R^{2}\right)$ better than 0.99 . The optimized kinetic parameters for each reaction step for the thermal decompositions of the purified palygorskite and sepiolite are summarized in Table 1. The contributions for the first reaction step, attributed to the thermal dehydration of zeolite water, were comparable between the two samples. This was also true for the thermal dehydration of the second coordinated water, which appeared as the fourth reaction step for the thermal decompositions of palygorskite and sepiolite. The optimized $E_{\mathrm{a}}$ values for each reaction step did not significantly change from the initial values in both samples. The rate behavior of each reaction step was simulated from the $\mathrm{SB}\left(m_{i}, n_{i}, p_{i}\right)$ model with the optimized kinetic exponents, as illustrated in Figure 8. The first reaction step, i.e., the thermal dehydration of zeolite water, exhibits nearly linear deceleration in both samples. The linear deceleration behavior was also seen for the third reaction step of the thermal decomposition of sepiolite. The corresponding reaction step for the thermal dehydration of the first coordinated water in the thermal decomposition of palygorskite exhibited zero-order-like behavior in a wide $\alpha_{3}$ range. For the other reaction steps in both samples, deceleration behavior characterized by concaved shapes was observed, possibly indicating that the process was controlled by diffusional removal of evolved water vapor.
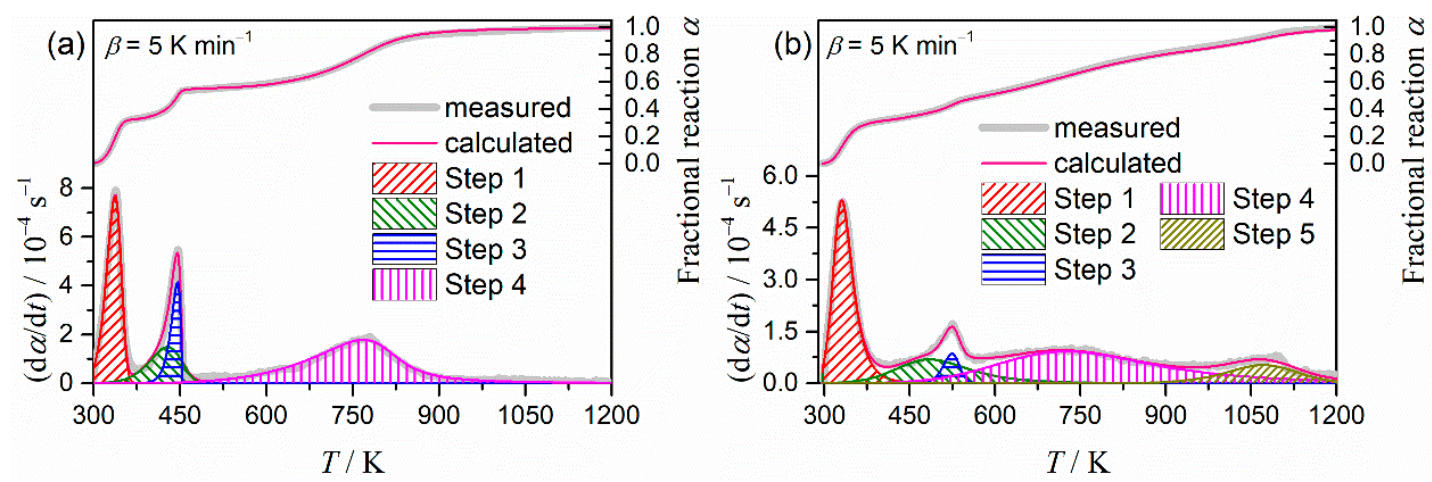

Figure 7. Typical results of KDA for the thermal decomposition of the purified clay minerals: (a) Palygorskite and (b) sepiolite. 
Table 1. The average kinetic parameters optimized by KDA for each reaction step of the thermal decomposition of the purified clay minerals.

\begin{tabular}{|c|c|c|c|c|c|c|c|c|}
\hline \multirow{2}{*}{ Sample } & \multirow{2}{*}{$i$} & \multirow{2}{*}{$c_{i}$} & \multirow{2}{*}{$E_{\mathrm{a}, i} / \mathrm{kJ} \mathrm{mol}^{-1}$} & \multirow{2}{*}{$A_{i} / \mathrm{s}^{-1}$} & \multicolumn{3}{|c|}{$f_{i}\left(\alpha_{i}\right)=\alpha_{i}^{m}\left(1-\alpha_{i}\right)^{n}\left[-\ln \left(1-\alpha_{i}\right)\right]^{p}$} & \multirow{2}{*}{$R^{2}$} \\
\hline & & & & & $m$ & $n$ & $p$ & \\
\hline \multirow{4}{*}{ Palygorskite } & 1 & $0.32 \pm 0.01$ & $63.7 \pm 0.2$ & $(4.35 \pm 0.07) \times 10^{7}$ & $-0.63 \pm 0.04$ & $1.26 \pm 0.03$ & $0.73 \pm 0.03$ & \multirow{4}{*}{$0.99 \pm 0.01$} \\
\hline & 2 & $0.12 \pm 0.02$ & $90.5 \pm 1.7$ & $(2.94 \pm 0.01) \times 10^{8}$ & $-0.33 \pm 0.02$ & $1.13 \pm 0.09$ & $-0.35 \pm 0.02$ & \\
\hline & 3 & $0.11 \pm 0.01$ & $114.4 \pm 0.2$ & $(2.00 \pm 0.02) \times 10^{11}$ & $0.03 \pm 0.01$ & $0.61 \pm 0.07$ & $0.21 \pm 0.01$ & \\
\hline & 4 & $0.45 \pm 0.01$ & $190.0 \pm 1.5$ & $(4.79 \pm 0.05) \times 10^{8}$ & $-32.8 \pm 3.8$ & $13.8 \pm 1.7$ & $29.5 \pm 3.7$ & \\
\hline \multirow{5}{*}{ Sepiolite } & 1 & $0.31 \pm 0.01$ & $80.4 \pm 0.1$ & $(3.25 \pm 0.02) \times 10^{10}$ & $0.33 \pm 0.02$ & $2.34 \pm 0.04$ & $-0.27 \pm 0.01$ & \multirow{5}{*}{$0.99 \pm 0.01$} \\
\hline & 2 & $0.14 \pm 0.01$ & $64.6 \pm 0.9$ & $(1.28 \pm 0.01) \times 10^{4}$ & $-0.01 \pm 0.01$ & $2.30 \pm 0.05$ & $-0.59 \pm 0.01$ & \\
\hline & 3 & $0.04 \pm 0.01$ & $213.0 \pm 1.0$ & $(1.01 \pm 0.01) \times 10^{19}$ & $-0.02 \pm 0.01$ & $1.41 \pm 0.02$ & $-0.09 \pm 0.01$ & \\
\hline & 4 & $0.41 \pm 0.01$ & $124.3 \pm 1.4$ & $(2.37 \pm 0.01) \times 10^{5}$ & $-0.46 \pm 0.01$ & $2.93 \pm 0.05$ & $-1.43 \pm 0.02$ & \\
\hline & 5 & $0.10 \pm 0.01$ & $738.4 \pm 1.6$ & $(9.50 \pm 0.01) \times 10^{32}$ & $-1.29 \pm 0.01$ & $2.30 \pm 0.01$ & $-1.78 \pm 0.02$ & \\
\hline
\end{tabular}



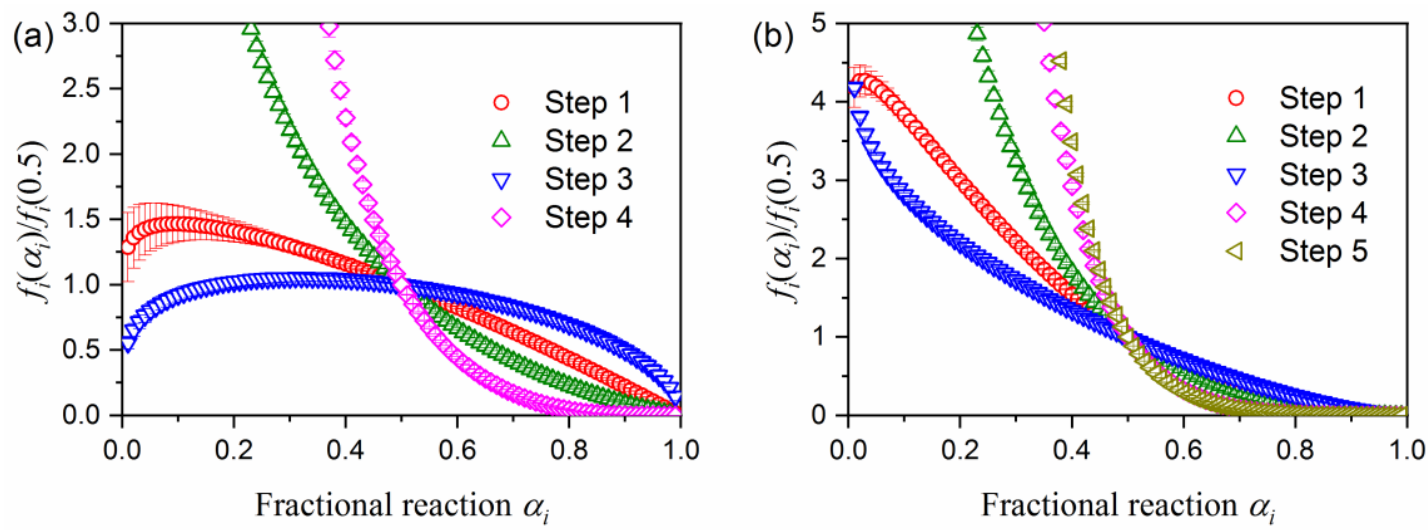

Figure 8. Rate behaviors for each reaction step of the thermal decomposition of (a) palygorskite and (b) sepiolite, reproduced from SB $\left(m_{i}, n_{i}, p_{i}\right)$ optimized by KDA.

\subsection{Kinetic Deconvolution Analysis for the Thermal Decomposition of $M B$}

Figure 9 presents the TG-DTG curves recorded at different $\beta$ values in a flow of air for the thermal decomposition of the synthesized P-MB and S-MB samples. The number of distinguishable DTG peaks was 5 and 6 for the thermal decomposition of P-MB and S-MB samples, respectively. In comparison with the TG-DTG curves for the clay mineral substrates (Figure 4), the third and fifth distinguishable DTG peaks in the thermal decomposition of P-MB appeared in addition to those expected from the thermal decomposition of palygorskite. For S-MB, the third and fourth distinguishable DTG peaks were the additional peaks. These additional peaks can be interpreted as the sublimation/decomposition of indigo incorporated into the clay mineral substrates.
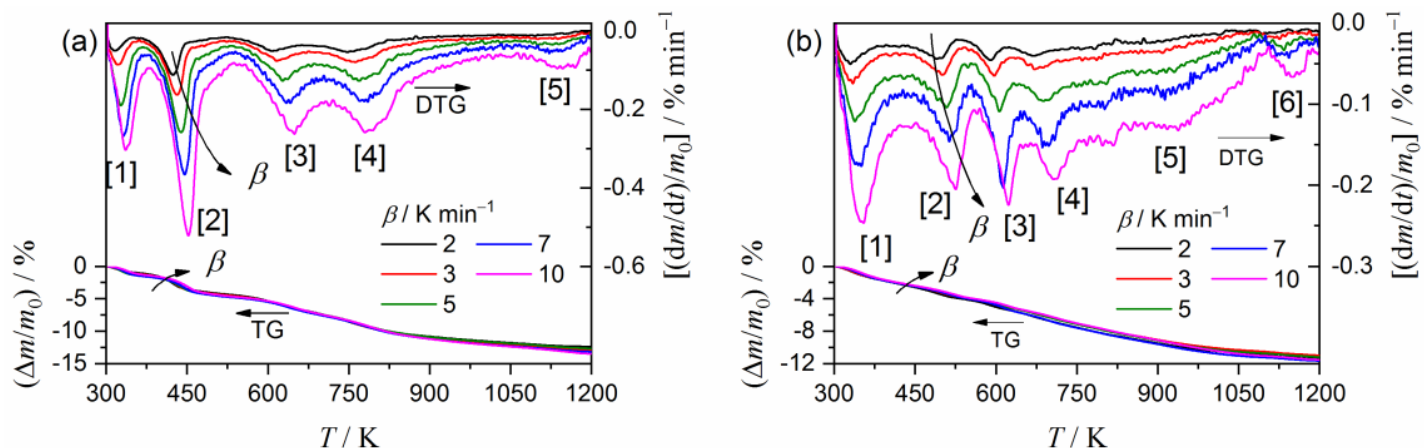

Figure 9. TG-DTG curves for the thermal decomposition of MB recorded at different $\beta$ in a flow of air: (a) P-MB and (b) S-MB.

MDA for the thermal decomposition of the MB samples was carried out by adding several minor peaks to the major discernable peaks in the DTG curves (Figure 9). Figure 10 illustrates typical results of MDA carried out by applying a Weibull function to each peak. By comparing the results of MDA for the thermal decomposition of clay mineral substrates, four additional peaks were revealed in both the P-MB and S-MB samples. These additional peaks (that is, the fourth, fifth, seventh, and eighth peaks for P-MB and the fourth, sixth, seventh, and eighth peaks for S-MB) are attributed to the thermal decomposition of the indigo molecules incorporated into the clay mineral matrix. The details of the analysis of the mathematically separated peaks are provided in Section S3 in the Supplementary Materials. 

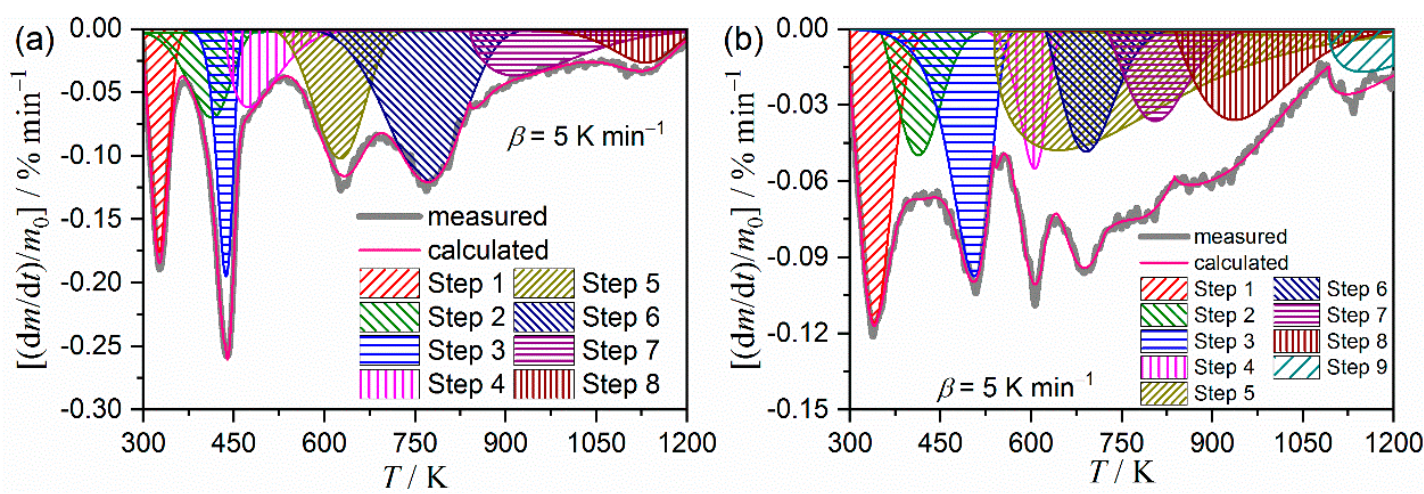

Figure 10. Typical results of MDA for the multistep thermal decomposition of MB samples: (a) P-MB and (b) S-MB.

Further, the overall thermal decomposition of the MB samples was analyzed by KDA. The initial values of $c_{i}$ were substituted in for the values determined by MDA (Table S2). For the reaction steps attributed to the thermal decomposition of the clay mineral substrates, the initial values of the kinetic parameters were substituted with values from the results of the KDA for thermal decomposition of the substrates (Table 1). For the reaction steps attributed to the thermal decomposition of indigo, the apparent $E_{\mathrm{a}, i}$ values determined for the corresponding steps using MDA (Table S2) were used as the initial values. The $f_{i}\left(\alpha_{i}\right)$ for the reaction steps of the thermal decomposition of indigo were set to be $\mathrm{SB}(0,1,0)$ as the initial setting. Then, the order of apparent $A_{i}$ values was determined by graphically comparing the fit of the calculated curves with the experimental kinetic curve.

Figure 11 illustrates typical results of KDA for the thermal decomposition of the MB samples. The nearly perfect fit using Equation (1) with $\mathrm{SB}(m, n, p)$ in Equation (5) as the kinetic model function was realized by using the optimized kinetic parameters for each reaction step, as listed in Table 2. The optimized kinetic parameters for each reaction step from the overall kinetic curves recorded at different $\beta$ values were practically invariant, as was determined by the acceptably small standard deviation values of each kinetic parameter. The sums of all the contributions from the reaction steps attributed to the thermal decomposition of indigo, that is, $i=4,5,7$, and 8 for the P-MB sample and $i=4,6,7$, and 8 for the S-MB sample, were 0.415 and 0.226 , respectively. Compared to the initial sample mass $m_{0}$, the fractional mass loss attributed to the thermal decomposition of indigo were calculated to be $5.35 \%$ and $2.58 \%$ for the P-MB and S-MB samples, respectively. These fractional mass-loss values are smaller than the initial mass ratio of indigo added to the clay mineral, $5.66 \%$, before heat treatment and purification, in both samples. The difference in the mass-loss fractions between both samples indicates that the P-MB sample incorporates twice as much indigo as the S-MB sample.
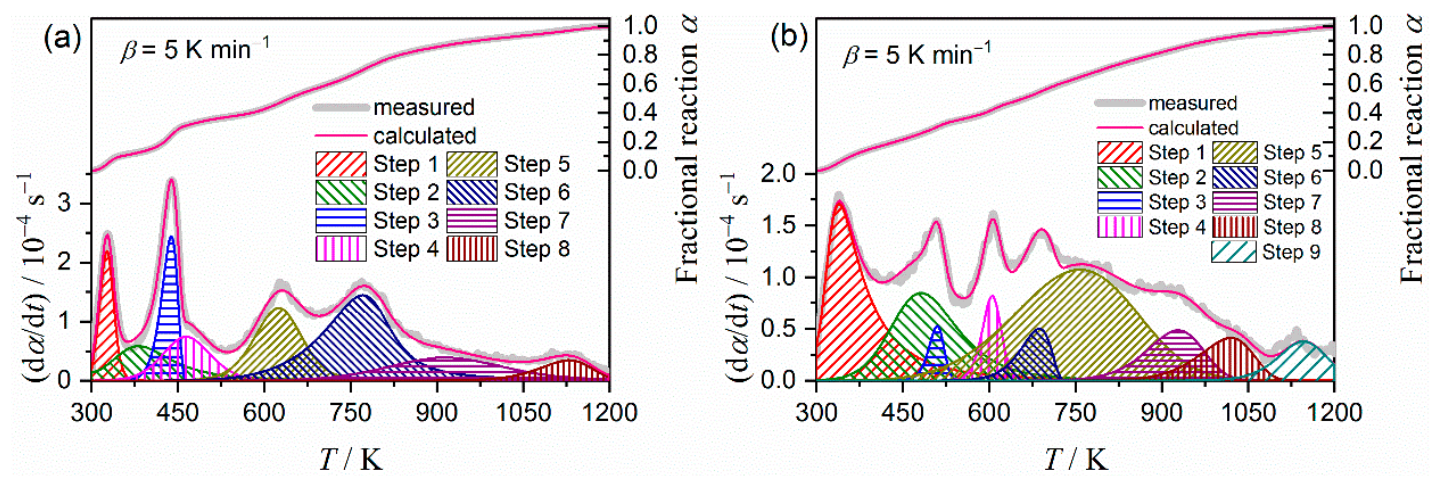

Figure 11. Typical results of KDA for the thermal decomposition of MB: (a) P-MB and (b) S-MB. 
Table 2. Kinetic parameters for each reaction step for the thermal decomposition of MB, as determined by KDA.

\begin{tabular}{|c|c|c|c|c|c|c|c|c|}
\hline \multirow{2}{*}{ Sample } & \multirow{2}{*}{$i$} & \multirow{2}{*}{$c_{i}$} & \multirow{2}{*}{$E_{\mathrm{a}, i} / \mathrm{kJ} \mathrm{mol}^{-1}$} & \multirow{2}{*}{$A_{i} / \mathrm{s}^{-1}$} & \multicolumn{3}{|c|}{$f_{i}\left(\alpha_{i}\right)=\alpha_{i}^{m}\left(1-\alpha_{i}\right)^{n}\left[-\ln \left(1-\alpha_{i}\right)\right]^{p}$} & \multirow{2}{*}{$R^{2}$} \\
\hline & & & & & $m$ & $n$ & $p$ & \\
\hline \multirow{8}{*}{ P-MB } & 1 & $0.08 \pm 0.01$ & $61.7 \pm 0.5$ & $(4.33 \pm 0.02) \times 10^{7}$ & $-0.57 \pm 0.10$ & $1.69 \pm 0.09$ & $0.73 \pm 0.05$ & \multirow{8}{*}{$0.99 \pm 0.01$} \\
\hline & 2 & $0.10 \pm 0.01$ & $46.4 \pm 0.3$ & $(1.95 \pm 0.01) \times 10^{3}$ & $-0.04 \pm 0.01$ & $2.68 \pm 0.22$ & $-0.18 \pm 0.01$ & \\
\hline & 3 & $0.10 \pm 0.01$ & $113.0 \pm 0.6$ & $(1.70 \pm 0.01) \times 10^{11}$ & $0.02 \pm 0.01$ & $0.85 \pm 0.01$ & $-0.07 \pm 0.01$ & \\
\hline & 4 & $0.09 \pm 0.01$ & $43.7 \pm 2.0$ & $(1.96 \pm 0.02) \times 10^{2}$ & $1.19 \pm 0.06$ & $1.05 \pm 0.03$ & $-1.10 \pm 0.05$ & \\
\hline & 5 & $0.15 \pm 0.03$ & $143.2 \pm 0.3$ & $(1.99 \pm 0.02) \times 10^{9}$ & $-0.11 \pm 0.01$ & $1.64 \pm 0.17$ & $-0.35 \pm 0.03$ & \\
\hline & 6 & $0.31 \pm 0.03$ & $183.2 \pm 2.5$ & $(4.99 \pm 0.03) \times 10^{8}$ & $-26.61 \pm 3.69$ & $11.94 \pm 2.27$ & $24.50 \pm 4.38$ & \\
\hline & 7 & $0.13 \pm 0.01$ & $634.3 \pm 3.6$ & $(1.70 \pm 0.01) \times 10^{32}$ & $-2.44 \pm 0.19$ & $3.79 \pm 0.57$ & $-3.82 \pm 0.43$ & \\
\hline & 8 & $0.05 \pm 0.01$ & $448.3 \pm 2.8$ & $(8.84 \pm 0.08) \times 10^{17}$ & $-0.39 \pm 0.03$ & $1.21 \pm 0.12$ & $-0.48 \pm 0.06$ & \\
\hline \multirow{9}{*}{ S-MB } & 1 & $0.22 \pm 0.01$ & $82.4 \pm 0.5$ & $(2.16 \pm 0.01) \times 10^{10}$ & $0.25 \pm 0.01$ & $5.57 \pm 0.09$ & $-0.35 \pm 0.01$ & \multirow{9}{*}{$0.98 \pm 0.02$} \\
\hline & 2 & $0.15 \pm 0.01$ & $64.5 \pm 0.8$ & $(1.29 \pm 0.01) \times 10^{4}$ & $-0.01 \pm 0.01$ & $2.45 \pm 0.08$ & $-0.40 \pm 0.01$ & \\
\hline & 3 & $0.02 \pm 0.01$ & $206.2 \pm 1.9$ & $(9.68 \pm 0.01) \times 10^{18}$ & $-0.02 \pm 0.01$ & $1.26 \pm 0.01$ & $-0.11 \pm 0.01$ & \\
\hline & 4 & $0.05 \pm 0.01$ & $160.3 \pm 0.4$ & $(4.48 \pm 0.02) \times 10^{11}$ & $0.06 \pm 0.01$ & $1.35 \pm 0.08$ & $0.24 \pm 0.01$ & \\
\hline & 5 & $0.34 \pm 0.01$ & $127.9 \pm 2.4$ & $(2.33 \pm 6.98) \times 10^{5}$ & $-0.46 \pm 0.01$ & $1.17 \pm 0.02$ & $-1.39 \pm 0.05$ & \\
\hline & 6 & $0.04 \pm 0.01$ & $180.6 \pm 0.7$ & $(1.36 \pm 0.01) \times 10^{11}$ & $-0.20 \pm 0.01$ & $0.78 \pm 0.01$ & $-0.20 \pm 0.01$ & \\
\hline & 7 & $0.08 \pm 0.01$ & $120.8 \pm 3.3$ & $(9.94 \pm 0.02) \times 10^{3}$ & $0.06 \pm 0.01$ & $1.07 \pm 0.01$ & $0.12 \pm 0.01$ & \\
\hline & 8 & $0.06 \pm 0.01$ & $314.0 \pm 5.5$ & $(1.51 \pm 0.01) \times 10^{13}$ & $-0.40 \pm 0.01$ & $1.09 \pm 0.01$ & $-0.33 \pm 0.01$ & \\
\hline & 9 & $0.05 \pm 0.01$ & $780.9 \pm 9.1$ & $(9.65 \pm 0.01) \times 10^{32}$ & $-0.73 \pm 0.01$ & $2.14 \pm 0.03$ & $-0.66 \pm 0.01$ & \\
\hline
\end{tabular}


Figure 12 presents typical optical microscopic views of the P-MB sample as it was heated to different temperatures at $\beta=10 \mathrm{~K} \mathrm{~min}^{-1}$ in flowing air. The brilliant blue color of the original P-MB (Figure 12a) was maintained until the sample reached $573 \mathrm{~K}$ (Figure 12b), which is a higher temperature than the completion of the fourth reaction step, i.e., the first reaction step of the thermal decomposition of the indigo. This observation supports our previous assumption that the first decomposition step of indigo is the sublimation/decomposition of indigo adsorbed on the surface of the clay mineral substrate, not the indigo incorporated into the clay mineral matrix. Color degradation was observed in the temperature range that corresponds to the fifth reaction step, which is the second decomposition step of incorporated indigo (Figure 12b-d). Thus, the second decomposition step of indigo was interpreted as the decomposition of indigo incorporated into the micropores of the clay mineral substrate. The grayish color of the sample after the fifth reaction step was completed (Figure 12d) was due to the products of indigo decomposition. Upon further heating, the grayish color gradually disappeared after the seventh and eighth reaction steps (Figure 12e,f, respectively), which corresponded to the third and fourth decomposition steps of indigo, respectively. This observation was understood to be the oxidative decomposition of the residues in the flowing air atmosphere.

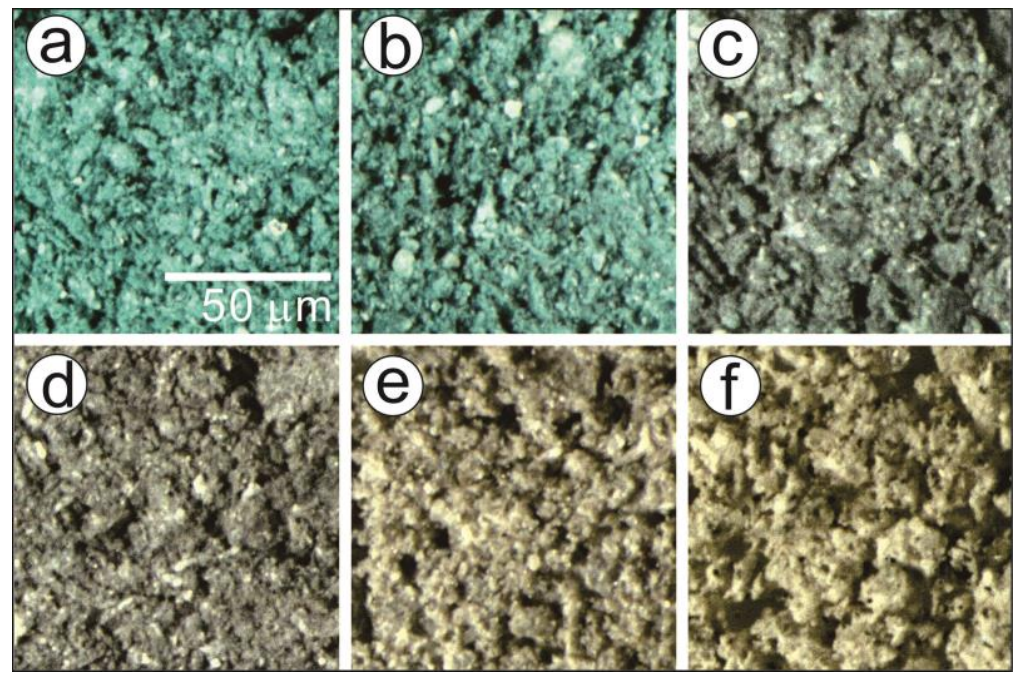

Figure 12. Typical optical microscopic views of the P-MB heated to different temperatures: (a) original sample, (b) $573 \mathrm{~K}$, (c) $713 \mathrm{~K}$, (d) $793 \mathrm{~K}$, (e) $1003 \mathrm{~K}$, and (f) $1223 \mathrm{~K}$.

To compare the thermal decomposition behavior between the indigo incorporated into the $\mathrm{MB}$ and pure indigo crystals, thermally induced changes in pure indigo were subjected to a formal kinetic study. Approximately $97.8 \pm 0.1 \%$ of mass loss was observed during the thermally induced sublimation/decomposition of the pure indigo crystals. The residue $(2.2 \%)$ was lost by the oxidative decomposition at a higher temperature. The sublimation/decomposition process was characterized by a constant $E_{\mathrm{a}}$ value of $150.7 \pm 4.7 \mathrm{~kJ} \mathrm{~mol}^{-1}$ and a phase-boundary controlled model. The details of the kinetic analysis are described in Section S4 in the Supplementary Materials.

Figure 13 compares the extracted kinetic curve $\left(\beta=5 \mathrm{~K} \mathrm{~min}^{-1}\right)$ and Arrhenius plots for the respective reaction steps, drawn using the optimized Arrhenius parameters listed in Table 2 for the thermal decomposition of indigo incorporated in P-MB $(i=5)$ and S-MB $(i=6)$, with those for the thermally induced sublimation/decomposition of pure indigo crystals. Although the thermal decomposition of indigo in P-MB starts at roughly the same temperature, the reaction proceeds at a slower rate and continues to higher temperatures in comparison to the pure indigo crystals (Figure 13a). Despite the differences in the kinetic data, the Arrhenius plots for these reactions are comparable (Figure 13b), as follows: $\left(E_{\mathrm{a}} / \mathrm{kJ} \mathrm{mol}^{-1}, A / \mathrm{s}^{-1}\right)$ values for P-MB $(i=5)$ are $\left(143.2 \pm 0.3,(1.99 \pm 0.02) \times 10^{9}\right)$. Mechanistic differences are a possible reason for the different kinetic behaviors. By contrast, the kinetic data and the Arrhenius plot for the thermal decomposition of the indigo incorporated into S-MB are 
very different from those of other indigo, having a higher thermal stability and a slower reaction rate. The larger Arrhenius parameters for S-MB $(i=6)$, i.e., $\left(180.6 \pm 0.7,(1.36 \pm 0.01) \times 10^{11}\right)$, explain the difference between its thermal behavior and that of P-MB.
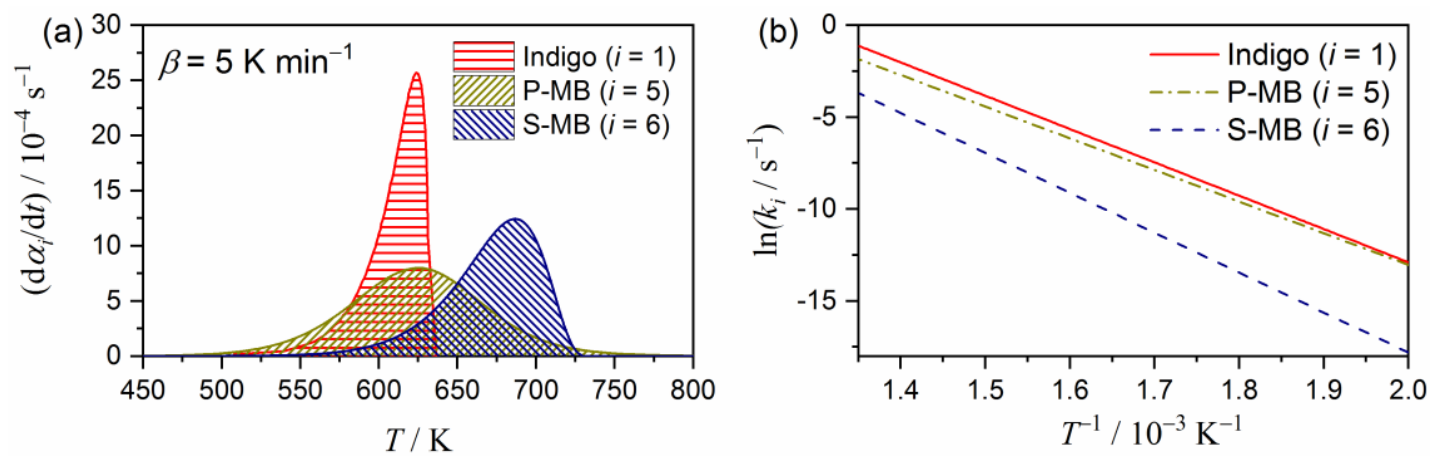

Figure 13. Comparison of the kinetic characteristics for the thermally induced sublimation/ decomposition of indigo as crystalline particles, incorporated in P-MB $(i=5)$, and in S-MB $(i=$ 6): (a) Kinetic curves and (b) Arrhenius plots.

Figure 14 is the plot of $f_{i}\left(\alpha_{i}\right)$ (= $\left.\mathrm{SB}\left(m_{i}, n_{i}, p_{i}\right)\right)$ versus $\alpha_{i}$ for the thermal decomposition step of indigo incorporated into the pores of the substrate minerals, i.e., P-MB $(i=5)$ and S-MB $(i=6)$. In both samples, the curves exhibit a concaved shape, which is characteristic of the deceleration process being controlled by diffusion. The curve was empirically fitted by a model for nucleation and growth controlled by diffusion, i.e., JMA $(m)$ with $m<1$ [55-58], or for three-dimensional shrinkage of reactant particle controlled by diffusion, i.e., the Jander model, D(3) [59].

$$
\begin{gathered}
\operatorname{JMA}(m): f(\alpha)=m(1-\alpha)[-\ln (1-\alpha)]^{1-1 / m} \\
\mathrm{D}(3): f(\alpha)=\frac{3(1-\alpha)^{2 / 3}}{2\left[1-(1-\alpha)^{1 / 3}\right]}
\end{gathered}
$$

The removal of gaseous products, which include sublimated indigo, by diffusion from the pores of the substrate mineral is likely the rate-limiting step. A reaction mechanism that is controlled by diffusion is very different from that of the sublimation/decomposition of pure indigo crystals (Figure S22c), which is also one reason for the thermal stability of the indigo incorporated into MB samples.
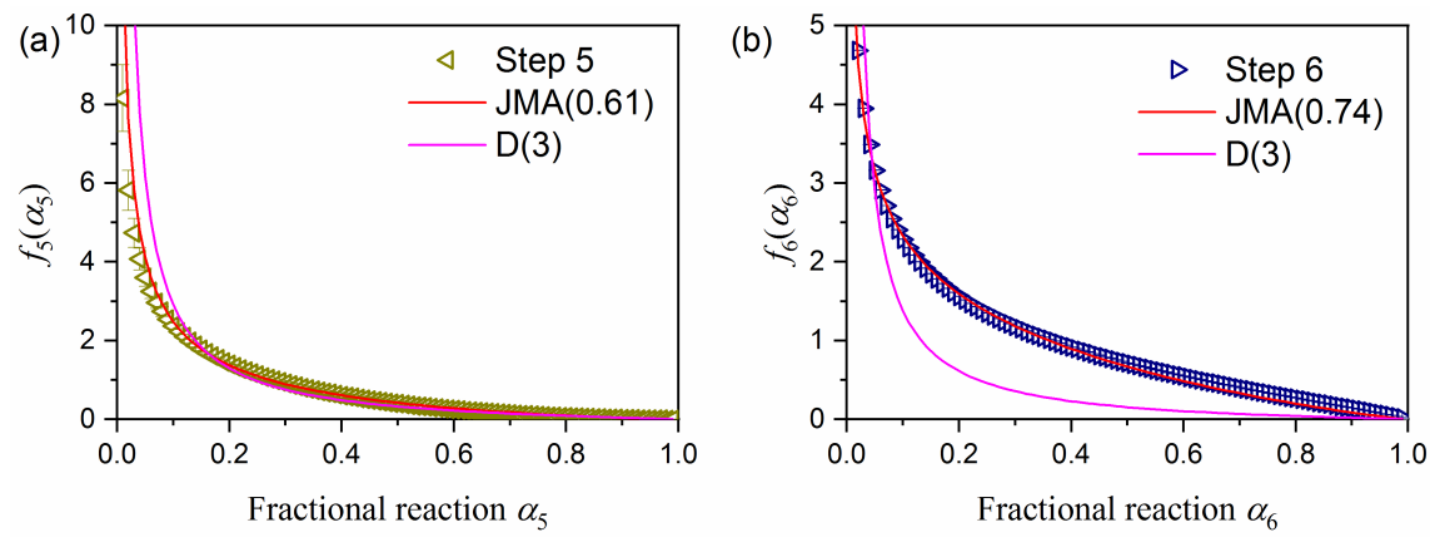

Figure 14. The plot of $f_{i}\left(\alpha_{i}\right)\left(=\mathrm{SB}\left(m_{i}, n_{i}, p_{i}\right)\right)$ versus $\alpha_{i}$ for the thermal decomposition step of indigo incorporated into the pores of the MB samples: (a) P-MB $(i=5)$ and $(\mathbf{b})$ S-MB $(i=6)$. 


\section{Materials and Methods}

\subsection{Sample Preparation}

The fibrous clay minerals, i.e., palygorskite and sepiolite, were purchased from SEPIO Japan. Each clay mineral was ground using an agate mortar and a pestle. The ground sample was sieved to different particle sizes using a series of sieves with different mesh sizes and an electric shaking apparatus (MVS-1, AS ONE). The sample particles sieved to 170-200 $\mu \mathrm{m}$ in diameter were used to synthesize $\mathrm{MB}$. The clay mineral samples were immersed in $1 \mathrm{M}-\mathrm{HCl}(\mathrm{aq})$ and the slurry was stirred for approximately $10 \mathrm{~h}$ to remove the $\mathrm{CaCO}_{3}$ impurity included in the clay mineral [60]. The precipitate was filtered and washed repeatedly until the chloride ions were not detected in the filtrates against $\mathrm{AgNO}_{3}(\mathrm{aq})$. The separated clay minerals were dried in an electric oven (DK240S, YAMATO) at $343 \mathrm{~K}$ for $24 \mathrm{~h}$.

MB was prepared following previously reported procedures [31,33,35,40,41,43,61]. The clay minerals treated with $\mathrm{HCl}(\mathrm{aq})$ were used as the substrates for synthesizing $\mathrm{MB}$. Indigo ( $\geq 95.0 \%$, NACALAI Tesque) was used as the pigment. Indigo and the clay mineral substrate were mixed in the following mass ratios: $0.01,0.02,0.06$, or 0.10 . Approximately $2.5 \mathrm{~g}$ of the mixed sample was placed into a ceramic crucible and covered with a ceramic lid. Samples were heated in an electric furnace (KDF P-70, DENKEN) at $368 \mathrm{~K}$ for $24 \mathrm{~h}$ and subsequently at $413 \mathrm{~K}$ for $24 \mathrm{~h}$.

Excess indigo that was not reacted with the clay mineral substrate was removed as follows [62]. Approximately $0.5 \mathrm{~g}$ of the heat-treated sample was dispersed into $20 \mathrm{~mL}$ of a $0.25 \mathrm{M}-\mathrm{NaOH}(\mathrm{aq})$ solution, to which $0.5 \mathrm{~g}$ of sodium dithionate $\left(\mathrm{Na}_{2} \mathrm{~S}_{2} \mathrm{O}_{4}\right)$ had been dissolved. The samples were kept at $348 \mathrm{~K}$ and stirred using a magnetic stirrer for $5 \mathrm{~min}$. After filtration, the separated solid was washed repeatedly with water and dried in air.

\subsection{Sample Characterization}

The clay mineral samples were identified using powder X-ray diffractometry (XRD) and FT-IR. Samples were press-fitted to sample holders to carry out XRD measurement on a diffractometer (RINT-2200V, Rigaku, Tokyo, Japan) with a radiation source $(\mathrm{Cu}-\mathrm{K} \alpha, 40 \mathrm{kV}, 20 \mathrm{~mA})$ in the $2 \theta$ range of $5-60^{\circ}$ at a scan speed of $4^{\circ} \mathrm{min}^{-1}$. FT-IR spectra were measured in a wavenumber range of $400-4600 \mathrm{~cm}^{-1}$ using the diffuse reflectance method in a spectrophotometer (FT-IR8400S, Shimadzu, Kyoto, Japan) after diluting the sample with $\mathrm{KBr}$. The clay mineral substrates and MB samples were subjected to simultaneous TG-DTA, UV-Vis spectroscopy, and morphological observation using a SEM. Approximately $10 \mathrm{mg}$ of each sample was weighed into a platinum pan (5 mm in diameter and $2.5 \mathrm{~mm}$ in height) and heated at a $\beta$ of $10 \mathrm{~K} \mathrm{~min}^{-1}$ from room temperature to $1223 \mathrm{~K}$ in flowing $\mathrm{N}_{2}$ (flowrate: $300 \mathrm{~cm}^{3} \mathrm{~min}^{-1}$ ) for recording TG-DTA curves using an instrument (STA7300, Hitachi High-Tech. Sci., Tokyo, Japan). The UV-Vis spectra of the samples press-fitted to a glass slide were recorded in a wavelength range of 400-700 nm using a spectrophotometer (V-560, JASCO, Tokyo, Japan) equipped with an integrating sphere. For SEM observations, the sample was coated with a thin Pt layer by sputtering (30 mA, 40 s, JFC-1600, JEOL, Tokyo, Japan) and observed using an instrument (JSM-6510, JEOL).

\subsection{Tracking of the Thermal Decomposition Process}

Thermal behaviors of the clay minerals received and those that were treated with $\mathrm{HCl}(\mathrm{aq})$ were investigated by TG/DTA-mass spectrometry (MS). Approximately $10 \mathrm{mg}$ of each sample was weighed into a platinum pan ( $5 \mathrm{~mm}$ in diameter and $2.5 \mathrm{~mm}$ in height) and TG-DTA measurements were carried out using an instrument (Thermoplus TG-8120, Rigaku). The sample was heated from room temperature to $1223 \mathrm{~K}$ in flowing $\mathrm{He}\left(200 \mathrm{~cm}^{3} \mathrm{~min}^{-1}\right)$. During the TG-DTA measurement, the outlet gas from the instrument was transferred into a MS instrument (M-200QA, Anelva, Kanagawa, Japan) through a capillary tube $(0.007 \mathrm{~mm}$ in inner diameter and $0.8 \mathrm{~m}$ in length) that was heated at $500 \mathrm{~K}$. 
MS measurements (EMSN: 1.0 A; SEM: $1.0 \mathrm{kV}$ ) for the outlet gas were continuously repeated in a $\mathrm{m} / \mathrm{z}$ range of $10-50$.

Using the TG-DTA instrument (STA7300), approximately $10 \mathrm{mg}$ of MB samples were heated to different temperatures at $\beta=10 \mathrm{~K} \mathrm{~min}^{-1}$ in flowing air $\left(300 \mathrm{~cm}^{3} \mathrm{~min}^{-1}\right)$. After the sample was cooled to room temperature, the partially decomposed samples were observed using an optical microscope (SZX7, Olympus, Tokyo, Japan) for recording the color of the sample.

\subsection{Measurement of the Kinetic Data for the Thermal Decomposition}

To record the kinetic data for the thermal decomposition of the clay mineral substrates treated with $\mathrm{HCl}(\mathrm{aq})$ and the MB samples, TG-DTA measurements were carried out using the STA7300 instrument. Approximately $5 \mathrm{mg}$ of each sample was weighed into a platinum pan $(5 \mathrm{~mm}$ in diameter and $2.5 \mathrm{~mm}$ in height) and heated from room temperature to $1223 \mathrm{~K}$ at various $\beta$ values between 2 and $10 \mathrm{~K} \mathrm{~min}^{-1}$ in flowing $\mathrm{N}_{2}$ or air (flowrate: $300 \mathrm{~cm}^{3} \mathrm{~min}^{-1}$ ). The TG-DTA instrument was previously calibrated in view of mass change values and temperature using standard procedures.

\section{Conclusions}

Thermal decompositions of palygorskite and sepiolite were characterized by three and four distinguished DTG peaks, respectively, which were attributed to the dehydration of zeolite, coordinating, and structural water. Kinetically, the second mass-loss process in both palygorskite and sepiolite was further separated into two reaction steps. Consequently, the thermal decompositions of palygorskite and sepiolite were kinetically separated into four and five reaction steps, respectively. The processes occurring in $\mathrm{MB}$ samples, i.e., $\mathrm{P}-\mathrm{MB}$ and $\mathrm{S}-\mathrm{MB}$, were separated by KDA into eight and nine reaction steps, respectively. The additional four reaction steps that were observed for MB samples are attributed to the thermal decomposition of the indigo incorporated into the clay mineral substrates. Discoloration of MB samples occurred during the second reaction step of the thermal decomposition of the indigo incorporated into the clay mineral substrates. The second reaction step is expected to be directly correlated to the thermal stability of the MB samples as a pigment. The second step of the thermal decomposition of indigo in P-MB started at approximately the same temperature as the thermally induced sublimation/decomposition of pure indigo crystals. In addition, the apparent Arrhenius parameters evaluated for these reaction processes were also comparable. However, the second step in the thermal decomposition of indigo in P-MB occurs at a slower rate and continues to higher temperatures compared to pure indigo crystals. This is explained by different physico-geometrical reaction mechanisms; the thermally induced sublimation/decomposition of pure indigo crystals is a phase boundary-controlled reaction and the second reaction step of indigo decomposition in P-MB is a diffusion-controlled reaction. Even though the second reaction step of indigo decomposition in S-MB started at a higher temperature in comparison to that in P-MB, both reactions ended at around the same temperature. The difference in starting temperatures can be explained by the larger Arrhenius parameters for S-MB. Although the stability of MB is commonly discussed in connection with the strength of the chemical bonds between indigo and the clay mineral substrate, the present study indicates that the physico-geometrical kinetic behavior of the thermal decomposition of indigo incorporated into the clay mineral substrate is another important factor in discussing the thermal stability of MB.

Supplementary Materials: The following are available online, S1: Sample preparation and characterization (Figures S1-S13), S2: Kinetic analysis for the thermal decomposition of clay mineral substrate (Table S1, Figures S14-S16), S3: Kinetic analysis for the thermal decomposition of Maya blue (Table S2, Figures S17-S20), S4: Kinetic analysis for the thermally induced sublimation/decomposition of indigo (Figures S21 and S22).

Author Contributions: Conceptualization, N.K.; Methodology, N.K.; Software, N.K.; Formal Analysis, Y.Y.; Investigation, Y.Y.; Data Curation, Y.Y.; Writing-Original Draft Preparation, Y.Y.; Writing-Review \& Editing, N.K.; Visualization, Y.Y.; Supervision, N.K.; Project Administration, N.K.; Funding Acquisition, N.K.

Funding: This research was funded by JSPS KAKENHI grant number 17H00820. 
Conflicts of Interest: The authors declare no conflict of interest.

\section{References}

1. Galwey, A.K.; Brown, M.E. Thermal Decomposition of Ionic Solids; Elsevier: Amsterdam, The Netherlands, 1999; ISBN 9780444824370.

2. Galwey, A.K. Structure and order in thermal dehydrations of crystalline solids. Thermochim. Acta 2000, 355, 181-238. [CrossRef]

3. Koga, N.; Tanaka, H. A physico-geometric approach to the kinetics of solid-state reactions as exemplified by the thermal dehydration and decomposition of inorganic solids. Thermochim. Acta 2002, 388, 41-61. [CrossRef]

4. Kitabayashi, S.; Koga, N. Thermal decomposition of tin(II) oxyhydroxide and subsequent oxidation in air: Kinetic deconvolution of overlapping heterogeneous processes. J. Phys. Chem. C 2015, 119, 16188-16199. [CrossRef]

5. Nakano, M.; Wada, T.; Koga, N. Exothermic behavior of thermal decomposition of sodium percarbonate: Kinetic deconvolution of successive endothermic and exothermic processes. J. Phys. Chem. A 2015, 119, 9761-9769. [CrossRef]

6. Muravyev, N.V.; Koga, N.; Meerov, D.B.; Pivkina, A.N. Kinetic analysis of overlapping multistep thermal decomposition comprising exothermic and endothermic processes: Thermolysis of ammonium dinitramide. Phys. Chem. Chem. Phys. 2017, 19, 3254-3264. [CrossRef] [PubMed]

7. Koga, N.; Kameno, N.; Tsuboi, Y.; Fujiwara, T.; Nakano, M.; Nishikawa, K.; Iwasaki-Murata, A. Multistep thermal decomposition of granular sodium perborate tetrahydrate: A kinetic approach to complex reactions in solid-gas systems. Phys. Chem. Chem. Phys. 2018, 20, 12557-12573. [CrossRef] [PubMed]

8. Koga, N.; Kodani, S. Thermally induced carbonation of $\mathrm{Ca}(\mathrm{OH})_{2}$ in a $\mathrm{CO}_{2}$ atmosphere: Kinetic simulation of overlapping mass-loss and mass-gain processes in a solid-gas system. Phys. Chem. Chem. Phys. 2018, 20, 26173-26189. [CrossRef]

9. Noda, Y.; Koga, N. Phenomenological kinetics of the carbonation reaction of lithium hydroxide monohydrate: Role of surface product layer and possible existence of a liquid phase. J. Phys. Chem. C 2014, 118, 5424-5436. [CrossRef]

10. Koga, N.; Suzuki, Y.; Tatsuoka, T. Thermal dehydration of magnesium acetate tetrahydrate: Formation and in situ crystallization of anhydrous glass. J. Phys. Chem. B 2012, 116, 14477-14486. [CrossRef]

11. Koga, N.; Yamada, S.; Kimura, T. Thermal decomposition of silver carbonate: Phenomenology and physicogeometrical kinetics. J. Phys. Chem. C 2013, 117, 326-336. [CrossRef]

12. Wada, T.; Koga, N. Kinetics and mechanism of the thermal decomposition of sodium percarbonate: Role of the surface product layer. J. Phys. Chem. A 2013, 117, 1880-1889. [CrossRef] [PubMed]

13. Yoshikawa, M.; Yamada, S.; Koga, N. Phenomenological interpretation of the multistep thermal decomposition of silver carbonate to form silver metal. J. Phys. Chem. C 2014, 118, 8059-8070. [CrossRef]

14. Wada, T.; Nakano, M.; Koga, N. Multistep kinetic behavior of the thermal decomposition of granular sodium percarbonate: Hindrance effect of the outer surface layer. J. Phys. Chem. A 2015, 119, 9749-9760. [CrossRef] [PubMed]

15. Tsuboi, Y.; Koga, N. Thermal decomposition of biomineralized calcium carbonate: Correlation between the thermal behavior and structural characteristics of avian eggshell. ACS Sustainable Chem. Eng. 2018, 6, 5283-5295. [CrossRef]

16. Kameno, N.; Koga, N. Heterogeneous kinetic features of the overlapping thermal dehydration and melting of thermal energy storage material: Sodium thiosulfate pentahydrate. J. Phys. Chem. C 2018, 122, 8480-8490. [CrossRef]

17. Koga, N. Physico-Geometric Approach to the Kinetics of Overlapping Solid-State Reactions. In Handbook of Thermal Analysis and Calorimetry, 2nd ed.; Vyazovkin, S., Koga, N., Schick, C., Eds.; Elsevier: Amsterdam, The Netherlands, 2018; Volume 6, pp. 213-251. [CrossRef]

18. Koga, N.; Goshi, Y.; Yamada, S.; Pérez-Maqueda, L.A. Kinetic approach to partially overlapped thermal decomposition processes. J. Therm. Anal. Calorim. 2013, 111, 1463-1474. [CrossRef]

19. Sánchez-Jiménez, P.E.; Perejón, A.; Criado, J.M.; Diánez, M.J.; Pérez-Maqueda, L.A. Kinetic model for thermal dehydrochlorination of poly(vinyl chloride). Polymer 2010, 51, 3998-4007. [CrossRef] 
20. Kitabayashi, S.; Koga, N. Physico-geometrical mechanism and overall kinetics of thermally induced oxidative decomposition of tin(II) oxalate in air: Formation process of microstructural tin(IV) oxide. J. Phys. Chem. C 2014, 118, 17847-17861. [CrossRef]

21. Ogasawara, H.; Koga, N. Kinetic modeling for thermal dehydration of ferrous oxalate dihydrate polymorphs: A combined model for induction period-surface reaction-phase boundary reaction. J. Phys. Chem. A 2014, 118, 2401-2412. [CrossRef]

22. Fukuda, M.; Koga, N. Kinetics and mechanisms of the thermal decomposition of copper(II) hydroxide: A consecutive process comprising induction period, surface reaction, and phase boundary-controlled reaction. J Phys Chem C 2018, 122, 12869-12879. [CrossRef]

23. Nishikawa, K.; Ueta, Y.; Hara, D.; Yamada, S.; Koga, N. Kinetic characterization of multistep thermal oxidation of carbon/carbon composite in flowing air. J. Therm. Anal. Calorim. 2017, 128, 891-906. [CrossRef]

24. Hara, D.; Nishikawa, K.; Koga, N. Characterization of carbon/carbon composites by kinetic deconvolution analysis for a thermal oxidation process: An examination using a series of mechanical pencil leads. Ind. Eng. Chem. Res. 2018, 57, 14460-14469. [CrossRef]

25. Kikuchi, S.; Koga, N.; Yamazaki, A. Comparative study on the thermal behavior of structural concretes of sodium-cooled fast reactor. J. Therm. Anal. Calorim. 2019. [CrossRef]

26. Koga, N.; Kikuchi, S. Thermal behavior of perlite concrete used in a sodium-cooled fast reactor: Multistep reaction kinetics and melting for safety assessment. J. Therm. Anal. Calorim. 2019. [CrossRef]

27. Nakano, M.; Fujiwara, T.; Koga, N. Thermal decomposition of silver acetate: Physico-geometrical kinetic features and formation of silver nanoparticles. J. Phys. Chem. C 2016, 120, 8841-8854. [CrossRef]

28. Fujiwara, T.; Yoshikawa, M.; Koga, N. Kinetic approach to multistep thermal behavior of $\mathrm{Ag}_{2} \mathrm{CO}_{3}-$ graphite mixtures: Possible formation of intermediate solids with $\mathrm{Ag}_{2} \mathrm{O}-\mathrm{Ag}$ and $\mathrm{Ag}_{2} \mathrm{CO}_{3}-\mathrm{Ag}$ core-shell structures. Thermochim. Acta 2016, 644, 50-60. [CrossRef]

29. Koga, N.; Kasahara, D.; Kimura, T. Aragonite crystal growth and solid-state aragonite-calcite transformation: A physico-geometrical relationship via thermal dehydration of included water. Cryst. Growth Des. 2013, 13, 2238-2246. [CrossRef]

30. Koga, N.; Nishikawa, K. Mutual Relationship between solid-state aragonite-calcite transformation and thermal dehydration of included water in coral aragonite. Cryst. Growth Des. 2014, 14, 879-887. [CrossRef]

31. Polette-Niewold, L.A.; Manciu, F.S.; Torres, B.; Alvarado, M., Jr.; Chianelli, R.R. Organic/inorganic complex pigments: Ancient colors Maya blue. J. Inorg. Biochem. 2007, 101, 1958-1973. [CrossRef]

32. Giustetto, R.; Seenivasan, K.; Bordiga, S. Spectroscopic characterization of a sepiolite-based Maya blue pigment. Period. Mineral. 2010, 79, 21-37. [CrossRef]

33. Chiari, G.; Giustetto, R.; Druzik, J.; Doehne, E.; Ricchiardi, G. Pre-columbian nanotechnology: Reconciling the mysteries of the maya blue pigment. Appl. Phys. A 2007, 90, 3-7. [CrossRef]

34. Ovarlez, S.; Giulieri, F.; Delamare, F.; Sbirrazzuoli, N.; Chaze, A.-M. Indigo-sepiolite nanohybrids: Temperature-dependent synthesis of two complexes and comparison with indigo-palygorskite systems. Microporous Mesoporous Mater. 2011, 142, 371-380. [CrossRef]

35. Giustetto, R.; Seenivasan, K.; Bonino, F.; Ricchiardi, G.; Bordiga, S.; Chierotti, M.R.; Gobetto, R. Host/guest interactions in a sepiolite-based Maya blue pigment: A Spectroscopic Study. J. Phys. Chem. C 2011, 115, 16764-16776. [CrossRef]

36. Hubbard, B.; Kuang, W.; Moser, A.; Facey, G.A.; Detellier, C. Structural study of Maya blue: Textural, thermal and solidstate multinuclear magnetic resonance characterization of the palygorskite-indigo and sepiolite-indigo adducts. Clays Clay Miner. 2003, 51, 318-326. [CrossRef]

37. Sánchez-Ochoa, F.; Cocoletzi, G.H.; Canto, G. Trapping and diffusion of organic dyes inside of palygorskite clay: The ancient Maya blue pigment. Microporous Mesoporous Mater. 2017, 249, 111-117. [CrossRef]

38. Giustetto, R.; Wahyudi, O. Sorption of red dyes on palygorskite: Synthesis and stability of red/purple Mayan nanocomposites. Microporous Mesoporous Mater. 2011, 142, 221-235. [CrossRef]

39. Tartaglione, G.; Tabuani, D.; Camino, G. Thermal and morphological characterisation of organically modified sepiolite. Microporous Mesoporous Mater. 2008, 107, 161-168. [CrossRef]

40. Zhou, W.; Liu, H.; Xu, T.; Jin, Y.; Ding, S.; Chen, J. Insertion of isatin molecules into the nanostructure of palygorskite. RSC Adv. 2014, 4, 51978-51983. [CrossRef]

41. Giustetto, R.; Llabres, I.X.F.X.; Ricchiardi, G.; Bordiga, S.; Damin, A.; Gobetto, R.; Chierotti, M.R. Maya blue: A computational and spectroscopic study. J. Phys. Chem. B 2005, 109, 19360-19368. [CrossRef] 
42. Ovarlez, S.; Giulieri, F.; Chaze, A.M.; Delamare, F.; Raya, J.; Hirschinger, J. The incorporation of indigo molecules in sepiolite tunnels. Chem. Eur. J. 2009, 15, 11326-11332. [CrossRef]

43. Manciu, F.S.; Reza, L.; Polette, L.A.; Torres, B.; Chianelli, R.R. Raman and infrared studies of synthetic Maya pigments as a function of heating time and dye concentration. J. Raman Spectrosc. 2007, 38, 1193-1198. [CrossRef]

44. Ovarlez, S.; Chaze, A.-M.; Giulieri, F.; Delamare, F. Indigo chemisorption in sepiolite. Application to Maya blue formation. C. R. Chim. 2006, 9, 1243-1248. [CrossRef]

45. Tilocca, A.; Fois, E. The color and stability of Maya blue: TDDFT Calculations. J. Phys. Chem. C 2009, 113, 8683-8687. [CrossRef]

46. Post, J.E.; Heaney, P.J. Synchrotron powder X-ray diffraction study of the structure and dehydration behavior of palygorskite. Am. Mineral. 2008, 93, 667-675. [CrossRef]

47. Nagata, H. On dehydration of bound water of sepiolite. Clays Clay Miner. 1974, 22, 285-293. [CrossRef]

48. Koga, N.; Šesták, J.; Simon, P. Some Fundamental and Historical Aspects of Phenomenological Kinetics in the Solid State Studied by Thermal Analysis. In Thermal Analysis of Micro, Nano- and Non-Crystalline Materials; Šesták, J., Simon, P., Eds.; Springer: Dordrecht, The Netherlands, 2013; pp. 1-28.

49. Friedman, H.L. Kinetics of thermal degradation of cha-forming plastics from thermogravimetry, application to a phenolic plastic. J. Polym. Sci., Part C 1964, 6, 183-195. [CrossRef]

50. Perejón, A.; Sánchez-Jiménez, P.E.; Criado, J.M.; Pérez-Maqueda, L.A. Kinetic analysis of complex solid-state reactions. A new deconvolution procedure. J. Phys. Chem. B 2011, 115, 1780-1791. [CrossRef] [PubMed]

51. Svoboda, R.; Málek, J. Applicability of Fraser-Suzuki function in kinetic analysis of complex crystallization processes. J. Therm. Anal. Calorim. 2013, 111, 1045-1056. [CrossRef]

52. Šesták, J.; Berggren, G. Study of the kinetics of the mechanism of solid-state reactions at increasing temperatures. Thermochim. Acta 1971, 3, 1-12. [CrossRef]

53. Šesták, J. Diagnostic limits of phenomenological kinetic models introducing the accommodation function. J. Therm. Anal. 1990, 36, 1997-2007. [CrossRef]

54. Šesták, J. Rationale and fallacy of thermoanalytical kinetic patterns. J. Therm. Anal. Calorim. 2011, 110, 5-16. [CrossRef]

55. Avrami, M. Kinetics of phase change. I. General theory. J. Chem. Phys. 1939, 7, 1103-1112. [CrossRef]

56. Avrami, M. Kinetics of phase change. II. Transformation-time relations for random distribution of nuclei. J. Chem. Phys. 1940, 8, 212-223. [CrossRef]

57. Avrami, M. Kinetics of phase change. III. Granulation, phase change, and microstructure. J. Chem. Phys. 1941, 9, 177-184. [CrossRef]

58. Johnson, W.A.; Mehl, K.F. Reaction kinetics in processes of nucleation and growth. Trans. Am. Inst. Mining. Metall. Eng. 1939, 135, 416-458.

59. Jander, W. Reaktionen im festen zustande bei höheren temperaturen. Reaktionsgeschwindigkeiten endotherm verlaufender umsetzungen. Z. Anorg. Allg. Chem. 1927, 163, 1-30. [CrossRef]

60. Cui, W.; Zhang, H.; Xia, Y.; Zou, Y.; Xiang, C.; Chu, H.; Qiu, S.; Xu, F.; Sun, L. Preparation and thermophysical properties of a novel form-stable $\mathrm{CaCl}_{2} \cdot 6 \mathrm{H}_{2} \mathrm{O}$ /sepiolite composite phase change material for latent heat storage. J. Therm. Anal. Calorim. 2017, 131, 57-63. [CrossRef]

61. Leitão, I.M.V.; Seixas de Melo, J.S. Maya blue, an ancient guest-host pigment: Synthesis and models. J. Chem. Educ. 2013, 90, 1493-1497. [CrossRef]

62. Boykin, D.W. A convenient apparatus for small-scale dyeing with indigo. J. Chem. Educ. 1998, 75, 769. [CrossRef]

Sample Availability: Samples of the compounds are available from the authors. 